\title{
Diameter and photospheric structures of Canopus from AMBER/VLTI interferometry ${ }^{\star} \star \star$
}

\author{
A. Domiciano de Souza ${ }^{1}$, P. Bendjoya ${ }^{1}$, F. Vakili ${ }^{1}$, F. Millour ${ }^{2}$, and R. G. Petrov ${ }^{1}$ \\ 1 Lab. H. Fizeau, CNRS UMR 6525, Univ. de Nice-Sophia Antipolis, Obs. de la Côte d'Azur, Parc Valrose, 06108 Nice, France \\ e-mail: Armando.Domiciano@unice.fr \\ 2 Max-Planck-Institut für Radioastronomie, Auf dem Hügel 69, 53121 Bonn, Germany
}

Received 23 June 2008 / Accepted 25 July 2008

ABSTRACT

\begin{abstract}
Context. Direct measurements of fundamental parameters and photospheric structures of post-main-sequence intermediate-mass stars are required for a deeper understanding of their evolution.

Aims. Based on near-IR long-baseline interferometry we aim to resolve the stellar surface of the F0 supergiant star Canopus, and to precisely measure its angular diameter and related physical parameters.

Methods. We used the AMBER/VLTI instrument to record interferometric data on Canopus: visibilities and closure phases in the $H$ and $K$ bands with a spectral resolution of 35 . The available baselines $(\simeq 60-110 \mathrm{~m})$ and the high quality of the AMBER/VLTI observations allowed us to measure fringe visibilities as far as in the third visibility lobe.

Results. We determined an angular diameter of $\emptyset=6.93 \pm 0.15$ mas by adopting a linearly limb-darkened disk model. From this angular diameter and Hipparcos distance we derived a stellar radius $R=71.4 \pm 4.0 R_{\odot}$. Depending on bolometric fluxes existing in the literature, the measured $\varnothing$ provides two estimates of the effective temperature: $T_{\text {eff }}=7284 \pm 107 \mathrm{~K}$ and $T_{\text {eff }}=7582 \pm 252 \mathrm{~K}$.

Conclusions. In addition to providing the most precise angular diameter obtained to date, the AMBER interferometric data point towards additional photospheric structures on Canopus beyond the limb-darkened model alone. A promising explanation for such surface structures is the presence of convection cells. We checked such a hypothesis using first order star-cell models and concluded that the AMBER observations are compatible with the presence of surface convective structures. This direct detection of convective cells on Canopus from interferometry can provide strong constraints to radiation-hydrodynamics models of photospheres of F-type supergiants.
\end{abstract}

Key words. stars: fundamental parameters - stars: individual: Canopus - supergiants - methods: observational techniques: high angular resolution - techniques: interferometric

\section{Introduction}

The evolved star Canopus ( $\alpha$ Carinae, HD 45348) is the second brightest star $(V=-0.72)$ in the night sky, just after Sirius. Although Canopus is classified as a F0II star in the SIMBAD database, several works (e.g. Achmad et al. 1991; Desikachary \& Hearnshaw 1982) present it as a F0 supergiant (FOIb), which is more adapted to the measured luminosity of $L \simeq 13000-15000 L_{\odot}$ (e.g. Jerzykiewicz \& Molenda-Zakowicz 2000; Smiljanic et al. 2006).

During their evolution, intermediate-mass stars like Canopus $\left(M \simeq 10 M_{\odot}\right.$ ) leave the red giant branch $(\mathrm{RGB})$ and enter the blue-giant region, significantly increasing their effective temperature and performing the so-called blue-loop. Even if most stars found in the blue-giant region of the HR diagram are experiencing their blue-loop phase, there are still puzzling questions concerning this phase of stellar evolution (e.g. Xu \& Li 2004).

Another unresolved issue in the evolution of intermediatemass stars concerns some discrepancies between observed and predicted abundances. Additional internal mixing processes are invoked to explain the observations. Smiljanic et al. (2006)

\footnotetext{
* Based on observations performed at the European Southern Observatory, Chile under ESO Program 079.D-0507.

$\star \star$ Table 1 is only available in electronic form at

http://www . aanda.org
}

performed a detailed spectroscopic study of Canopus, and other intermediate-mass stars, in order to investigate the influence of rotation on the internal mixing of elements. They conclude that the abundance of many stars of their sample, including Canopus, are better explained by models including rotation effects in the evolutionary models.

To constrain evolutionary models it is crucial to have precise measurements of fundamental stellar parameters, such as the effective temperature $T_{\text {eff }}$, luminosity $L$, radius $R$. If the distance $d$ and the angular diameter $\emptyset$ are known, then a quite direct method to estimate $R$ is to apply the simple relation $R=0.5 \emptyset d$. The most direct and precise measurements of $\varnothing$ can be obtained by modern long baseline interferometers.

In this work we present a precise determination of the angular diameter (and other derived physical parameters) of Canopus from interferometric observations performed with the AMBER focal instrument (Petrov et al. 2007), installed at ESO-VLTI (Glindemann et al. 2004) located at Cerro Paranal, Chile. The observations and data reduction are described in Sects. 2 and 3. The results and conclusions are outlined in Sects. 4 and 5.

\section{Observations}

The AMBER observations were obtained at low spectral resolution $R_{\mathrm{S}}=\lambda / \Delta \lambda \simeq 35$ in the $H$ and $K$ bands (LR-HK mode), 


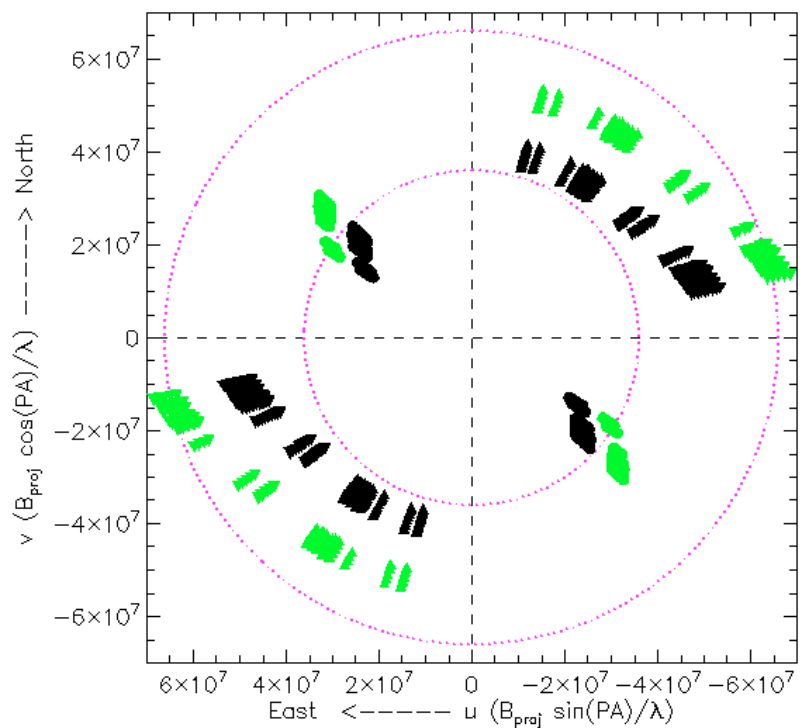

Fig. 1. The $u v$-plane coverage of the AMBER/VLTI observations of Canopus spanning the $H$ (green symbols) and $K$ (black symbols) bands with a spectral resolution of 35 . As a reference the dotted circles indicate the expected positions of the first and second visibility minima. Spatial frequencies range from the first to the third visibility lobes.

using three of the $2 \mathrm{~m}$-class Auxiliary Telescopes (ATs) placed at the VLTI stations A0, K0, and G1.

A relatively complete uv-plane coverage was obtained thanks to observations performed over 3 nights (2007 April 6-8) spanning $\simeq 2 \mathrm{~h}$ each night and several projected baselines ranging from $\simeq 60 \mathrm{~m}$ to $\simeq 110 \mathrm{~m}$ (Fig. 1). Each AMBER observing file consists of 1000 frames (one interference and three photometric channels) recorded with an integration time of $0.026 \mathrm{~s}$. A complete AMBER observing block is typically composed of 5 or 10 observing files recorded on the target followed by similar observations of the calibrator (HD 79917). These files allow the measurement of one set of calibrated squared visibilities (one at each baseline) and closure phases in the $H$ and $K$ bands. Further details on the observations are given in the online Table 1.

\section{Data reduction}

The data was reduced using the standard routines of the AMBER Data Reduction Software (called amdlib) version $2.1^{1}$. Tatulli et al. (2007) describe the principles of the AMBER-DRS routines allowing the conversion of raw-data frames into individual complex visibilities.

The best individual complex visibilities of an observing block are then selected and averaged to obtain uncalibrated visibilities and closure phases. Data selection is necessary since in the current version of AMBER-DRS no correction of the nonzero optical path difference (OPD) effect is applied. The determination of the selection parameters is thus an important step in the data reduction. Several tests indicated that good and robust results are obtained for the Canopus data when the following combination of selection parameters is adopted in the AMBER-DRS v2.1: flux SNR above 5, estimated absolute piston smaller than $10 \mu \mathrm{m}$, fringe SNR above 5 ( $K$ band) and above 3 ( $H$ band). We checked that this selection gives results compatible (within the error bars) with a previous version of

\footnotetext{
1 Available at http://www.jmmc.fr/data_processing_amber. htm
}

AMBER-DRS (amdlib v1.21) combined with similar selection procedures developed by us, as they were not yet fully implemented in the mainstream software.

Calibrated visibilities $(V)$ and closure phases are then obtained from selected and averaged visibilities and closure phases of Canopus and the calibrator. The uncertainties associated with the calibrated visibilities provided by AMBER-DRS v2.1 correspond in general to $₫ 0.02 \mathrm{~V}$. These low values take into account, for example, fundamental noises and the uncertainty on the calibrator's angular diameter (Table 1), but do not include time variations of the atmospheric plus instrumental transfer function of AMBER/VLTI. Thus, for each observing night we estimated this additional visibility uncertainty $\left(\sigma_{V}(\lambda)\right)$ from the several observations of the calibrator, averaging over the three baselines. Depending on the night and on the wavelength $\lambda$, the relative uncertainty was estimated as $\sigma_{V}(\lambda) / V(\lambda) \simeq 0.03-0.09$ in the $H$ band and $\sigma_{V}(\lambda) / V(\lambda) \simeq 0.02-0.06$ in the $K$ band. This additional $\sigma_{V}(\lambda)$ was quadratically added to the corresponding uncertainties from AMBER-DRS v2.1.

We investigated the presence of correlated noise in the AMBER data (Li Causi et al. 2008) as all data taken before September 2007 were affected by an electromagnetic interference producing spurious fringes on the detector array. These spurious fringes were corrupting the interferometric data quality. The AMDC software was developed by Li Causi et al. ${ }^{2}$ to filter out these spurious fringes. We applied the AMDC software on select observing files to check whether this procedure changes significantly the interferometric observables measured on Canopus. We found that, since the star is very bright, the correlated noise does not affect the observations in the range of their statistical error bars (relative differences are of the order of $10^{-3}$ ). Therefore, we neglect this step in all our data processing.

Currently, there is no standard procedure dedicated to the spectral calibration for AMBER, which introduces an uncertainty on the wavelength $\lambda$. We could partially correct this effect by comparing Earth atmospheric transmission curves with the AMBER/VLTI low resolution spectra of Canoups recorded in the $J, H$, and $K$ bands. From the positions of the strong atmospheric absorption features between the $J$ and $H$, and $H$ and $K$ bands we could determine that the original $\lambda^{\prime}$ given by AMBER should be corrected to new values $\lambda$ according to the equation $\lambda=\lambda^{\prime}+\delta \lambda$, where $\delta \lambda=-0.08 \mu \mathrm{m}$. Other possibilities to correct $\lambda$ exist, in particular we have also tried to determine this correction by directly fitting $\delta \lambda$ as a free parameter of our model. However, we found that the procedure using the atmospheric transmission gives more robust results. We also estimate that the uncertainty on the corrected wavelength $\sigma_{\lambda}$ is $\simeq 0.03 \mu \mathrm{m}$ ( $\simeq 1$ pixel on the detector). As shown in the next sections, such a relatively high value $\left(\sigma_{\lambda} \simeq 2 \% \lambda\right)$ has a direct consequence on the uncertainty of the measured angular sizes. These values agree with other corrections to the spectral calibration performed on different targets (Kraus, private comm.). A better spectral calibration procedure seems to be crucial to attain a higher precision in the measured angular sizes, especially in the LR mode.

Finally, we discarded the visibilities measured at the edges of the spectral bands because the data quality rapidly decreases in these regions. Hence, the results presented in the next sections include only wavelengths between $1.55 \mu \mathrm{m}$ and $1.7 \mu \mathrm{m}$ for the $H$ band, and between $2.0 \mu \mathrm{m}$ and $2.3 \mu \mathrm{m}$ for the $K$ band.

${ }^{2}$ Available at http://www mporzio.astro.it/ licausi/AMDC/ 

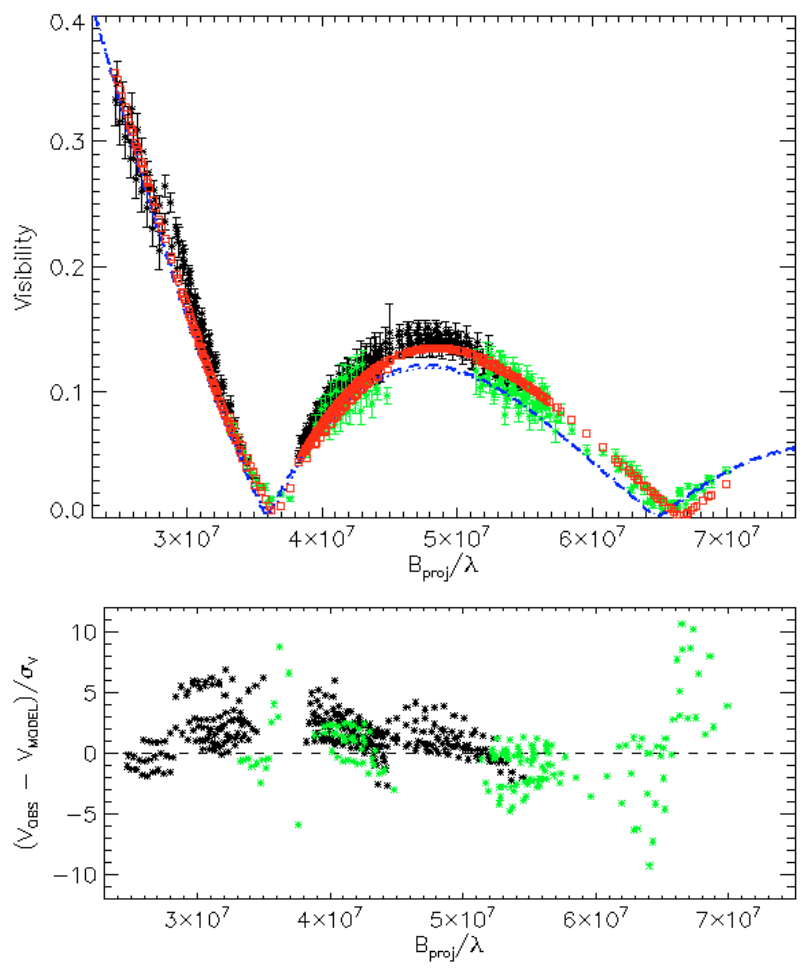

Fig. 2. Top: AMBER/VLTI visibility amplitudes and uncertainties in the $H$ (green) and $K$ bands (black). The model visibilities (red squares) were calculated with a linear limb-darkened disk model fitted to the data (Table 2). We also show the theoretical visibilities (dots for $H$ and dashes for $K$ ) expected for Canopus from a LLD disk with parameters determined by Claret (2000). Clearly these limb-darkened models do not account for the observations, especially after the first minimum. Bottom: fit residuals in $\sigma_{V}$ units showing a relatively important departure from zero, as well as residual trends indicating that additional effects should be included in the modeling of Canopus.

\section{Model fitting and physical parameters}

The observed visibilities (Fig. 2) and closure phases (Fig. 3) indicate that, as a zero-order approximation, Canopus can be considered to be a star having a centrally-symmetric intensity distribution (see also discussion in Sect. 5). We have thus fitted a linearly limb-darkened disk (LLD) model $^{3}$ to both $H$ and $K$ band observed visibilities. The free parameters of the model are the LLD angular diameter $\varnothing$, and the $H$ and $K$ band LLD coefficients $\epsilon_{H}$ and $\epsilon_{K}$. The estimated parameters and corresponding uncertainties obtained from a Levenberg-Marquardt (L-M) least-squares fit are given in Table 2. The model visibilities and fit residuals are shown in Fig. 2.

The uncertainty in $\varnothing\left(\sigma_{\varnothing}=2.2 \% \emptyset\right)$ is dominated by the uncertainty in the instrumental spectral calibration (cf. Sect. 3). Even with this limitation, AMBER/VLTI enabled us to derive the most precise angular diameter of Canopus known today. This interferometrically measured angular diameter is compatible with several previous measurements within the uncertainties, for example, $\varnothing=6.6 \pm 0.8$ mas (Hanbury-Brown et al. 1974; intensity interferometry), $\varnothing=7.00 \pm 0.41$ mas (Heras et al. 2002; spectral fit); additional values are listed by Heras et al. (2002).

In Table 2 we give the linear radius $R$ and effective temperature $T_{\text {eff }}$ derived from our angular diameter and other previously measured parameters. Thanks to the precision in $\emptyset$ attained with

\footnotetext{
${ }^{3} I(\mu) / I(1)=1-\epsilon(1-\mu)$, where $I$ is the specific intensity, and $\mu$ is the cos of the angle between the line of sight and the emergent intensity.
}

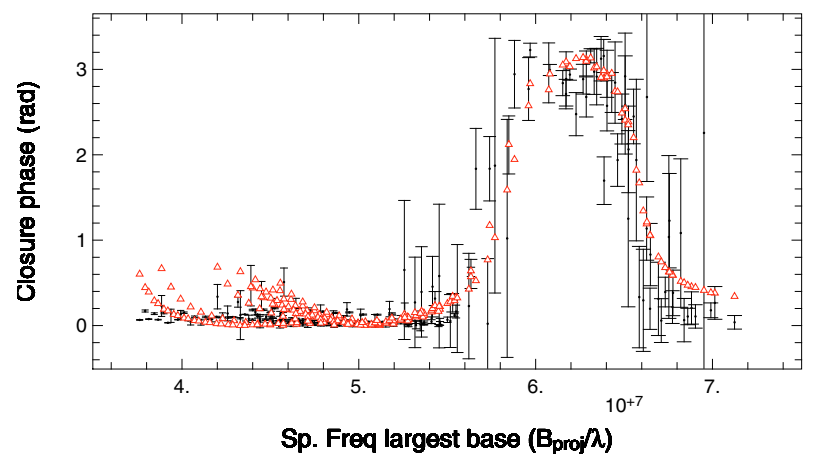

Fig. 3. AMBER/VLTI closure phases (absolute values) of Canopus as a function of the spatial frequency calculated on the longest baseline. Values indicate that Canopus does not have a completely centrally symmetric intensity distribution. For example the absolute values of the closure phases at low spatial frequencies $\left(\leq 5.5 \times 10^{7} \mathrm{cycles} / \mathrm{rad}\right)$ are of the order of $0.1-0.2 \mathrm{rad}$. The triangles indicate the theoretical closure phases obtained from the fit of a single convective cell model to the visibility amplitudes alone (see text for details).

Table 2. Parameters and uncertainties estimated from a least-square fit of a LLD disk model to the observed visibilities. The reduced $\chi^{2}$ of the fit is $\chi_{\text {red }}^{2}=7.0$, suggesting that the LLD disk cannot completely explain the observations. Physical parameters of Canopus derived from the measured $\varnothing$ are also listed.

\begin{tabular}{cc}
\hline \hline Fitted parameters & Derived parameters \\
\hline$\varnothing=6.93 \pm 0.15$ mas & $R / R_{\odot}=71.4 \pm 4.0^{a}$ \\
$\epsilon_{H}=0.04 \pm 0.01$ & $T_{\text {eff }}=7284 \pm 107 \mathrm{~K}^{b}$ or $7582 \pm 252 \mathrm{~K}^{c}$ \\
$\epsilon_{K}=-0.07 \pm 0.01$ & $\log g=1.7 \pm 0.1^{d}$ \\
\hline
\end{tabular}

${ }^{a}$ From the Hipparcos distance $d=95.9 \pm 4.9$ pc (Perryman et al. 1997). The Lutz-Kelker correction is negligible for Canopus (Jerzykiewicz \& Molenda-Zakowicz 2000).

${ }^{b}$ From the bolometric flux measured by Code et al. (1976): $f=$ $(45.0 \pm 1.8) \times 10^{-6} \mathrm{erg} \mathrm{cm}^{-2} \mathrm{~s}^{-1}$.

${ }^{c}$ Obtained by using values from Smiljanic et al. (2006) and references therein (visual apparent magnitude $V=-0.72$, extinction $A_{V}=0.08$, visual bolometric correction $B C_{V}=0.0$ ) and from Drilling \& Arlo (2000) (solar luminosity and bolometric magnitude). The uncertainty in $T_{\text {eff }}$ was estimated from $\sigma_{\Phi}$ and considering a conservative cumulative error of 0.1 in $V, A_{V}$, and $B C_{V}$.

${ }^{d}$ Assuming a mass of $M / M_{\odot}=9 \pm 2$.

AMBER, the uncertainty in $R$ is dominated by the uncertainty in the distance, contrarily to the previous measurements of $\emptyset$.

As indicated in Table 2, the two values of $T_{\text {eff }}$ were obtained from different approaches, corresponding to distinct estimates of the bolometric fluxes. One can find in the literature values compatible with both estimates. For example, Desikachary \& Hearnshaw (1982) and references therein give $T_{\text {eff }} \simeq 7300-7400 \mathrm{~K}$, while Kovtyukh (2007), Smiljanic et al. (2006) and references therein give $T_{\text {eff }} \simeq 7500-7600 \mathrm{~K}$.

Figure 2 shows that the near-IR intensity distribution of Canopus is not entirely compatible with a simple limb-darkened model. In particular the height of the second lobe is quite important, corresponding to $\epsilon_{H} \gtrsim 0$ and $\epsilon_{K} \lesssim 0$ (i.e., a slight limb-brightening). These measured values do not agree with the theoretical predictions of linear limb-darkening for Canopus. To illustrate this point we show in Fig. 2 the theoretical visibility curves for linear limb-darkening coefficients from Claret (2000): $\epsilon_{H}=0.28$ and $\epsilon_{K}=0.24$. These values correspond to $T_{\text {eff }}$ and $\log g$ from Table 2, and to the metallicity and microturbulence velocity from Smiljanic et al. (2006). 
Another discrepancy between observed and modeled visibilities is the fact that, after reaching its maximum, the second lobe decreases faster than the limb-darkened disk model; this effect can be better seen as a negative slope in the fit residuals. To investigate these effects in the second lobe we have also fitted the data using a LLD model with coefficients $\epsilon_{H}$ and $\epsilon_{K}$ varying linearly with $\lambda$, since the observations cover a relatively wide spectral range. However, no significantly better results were obtained.

In addition to the discrepancies mentioned above, it is crucial to note that the observed closure phases (Fig. 3) are incompatible with a centrally symmetric model such as a limb-darkened disk. All these observational evidences strongly suggest that there are other mechanisms at play on the surface of Canopus which induce asymmetric structures. We investigate this issue in the following section.

\section{Discussion and conclusions}

One promising explanation for the subtle interferometric signatures revealed by AMBER/VLTI is the presence of convective photospheric cells. These convective cells can introduce fine scale structures in the intensity maps that are detectable by stellar interferometry. Chiavassa (2008) uses 3D radiative transfer models to show that photospheric convection on red supergiants (RSG) can be detected by near-IR modern interferometers, already within the second visibility lobe (see for example the cover page of the EAS Publications Series 2008, vol. 28). The expected visibility signatures computed by Chiavassa are similar to those presented by the AMBER observations. Even if Canopus is closer to a yellow supergiant (YSG) than to a RSG, the presence of photospheric structures suggested by our observations is compatible with previous works revealing coronal (Brown et al. 2000; Westbrook et al. 2008) and chromospheric lines (Dupree et al. 2005), as well as a variable magnetic field (Weiss 1986; Bychkov et al. 2005) on this star. Additionally, during their blue loop phase, YSG can develop atmospheric instabilities when crossing different regions of $T_{\text {eff }}$ in the HR diagram (e.g. Achmad et al. 1991).

Freytag \& Ludwig (2007, FL07 hereafter) used a 3D radiation-hydrodynamics code to model convective structures on stellar atmospheres. For stars similar to Canopus $(\log g \simeq 1-2)$ they found granular cell sizes $\sim 1 \%-5 \%$ of the stellar diameter. Dravins (1990) studied the surface granulation of Canopus using a four-component parametrized model, concluding that $\sim 10 \%$ of the stellar surface is composed by hot rising granules, $\sim 20 \%$ by cool sinking gas, while the rest of Canopus surface remains quiescent. Based on these works suggesting the presence of photospheric structures on YSG we have attempted to explain the interferometric observations of Canopus by adopting simple exploratory models where the granular cells are mimicked by circles of uniform brightness added to a larger uniform disk representing the stellar photosphere itself with a total flux arbitrarily fixed at 1. Several L-M fits of the visibilities were performed leading to the following main conclusions:

1. Many-cells model: this model is based on the results from FL07 and corresponds to a regular grid of circles added to a uniform disk. The best fit is obtained for a cell diameter $\emptyset_{\text {cell }}=10 \% \varnothing$ and for a total individual cell flux of 0.07 . This model gives $\chi_{\text {red }}^{2}=7.0$, providing no improvement compared to the limb-darkened disk used in Sect. 4. By fixing $\varnothing_{\text {cell }}$ to $5 \% \varnothing$ (compatible with FL07) we obtain even worse results: $\chi_{\text {red }}^{2}=7.6$.

2. One-cell model: by fitting a single cell added to the stellar surface we obtain $\chi_{\text {red }}^{2}=4.0$ for $\emptyset_{\text {cell }}=36 \% \varnothing$ and a total cell flux of 0.035 . By fixing $\emptyset_{\text {cell }}$ to $5 \% \varnothing$ (compatible with FL07) we obtain $\chi_{\text {red }}^{2}=5.0$.

We note that we did not include the closure phases in the fits because they are not yet fully commissioned, especially regarding their error bars and their sign (which is why only absolute values are given in Fig. 3).

Results from these exploratory models indicate a general trend favoring large cell sizes compared to smaller ones. The best one-cell model $\left(\emptyset_{\text {cell }}=36 \% \emptyset\right)$ has a cell surface $\left(\emptyset_{\text {cell }}^{2}=\right.$ $13 \% \emptyset^{2}$ ) of similar order as the surface of the rising granules from the parametrized model of Dravins (1990): 10\%. The closure phases from this best-fit one-cell model agree well with the observed closure phases as shown in Fig. 3.

In this work we described and analyzed recent AMBER/VLTI observations of Canopus leading to the most precise angular diameter obtained to date, reaching a low relative uncertainty of $\simeq 2 \%$. Additionally, observations provide strong and direct support for the hypothesis of stellar granulation from convection cells, with an unexpected favoring of large cell sizes. A dedicated study of the surface granulation of Canopus would greatly benefit from a complete hydrodynamics and radiative transfer model revised for the Canopus case and from further aperture synthesis observations with AMBER/VLTI using several baselines and/or Earth-rotation.

Acknowledgements. This research used the SIMBAD and VIZIER databases at the CDS, Strasbourg (France), and NASA's ADS bibliographic services. We thank Dr. S. Kraus for his suggestions on the spectral calibration. F.M. acknowledges the Max-Planck-Institut (Bonn, Germany) for a postdoctoral fellowship.

\section{References}

Achmad, L., de Jager, C., \& Nieuwenhuijzen, H. 1991, A\&A, 249, 192 Brown, A., Ayres, T. R., Osten, R. A., \& Harper, G. M. 2000, BAAS, 32, 1257 Bychkov, V. D., Bychkova, L. V., \& Madej, J. 2005, A\&A, 430, 1143

Chiavassa, A. 2008, EAS Publications Series, ed. S. Wolf, F. Allard, \& Ph. Stee, 28,31

Claret, A. 2000, A\&A, 363, 1081

Code, A. D., Bless, R. C., Davis, J., \& Brown, R. H. 1976, ApJ, 203, 417

Desikachary, K., \& Hearnshaw, J. B. 1982, MNRAS, 201, 707

Dravins, D. 1990, A\&A, 228, 218

Drilling, J. S., \& Arlo, U. L. 2000, in Allen's Astrophysical Quantities, fourth edition, ed. A. N. Cox (Springer), 381

Dupree, A. K., Lobel, A., Young, P. R. et al. 2005, ApJ, 622, 629

Freytag, B., \& Ludwig, H.-G. 2007, Proc. SF2A, ed. J. Bouvier, A. Chalabaev, \& C. Charbonnel, 481

Glindemann, A., Albertsen, M., Andolfato, L., et al. 2004, Proc. SPIE, 5491, 447 Hanbury Brown, R., Davis, J., \& Allen, L. R. 1974, MNRAS, 167, 121 Heras, A. M., Shipman, R. F., Price, S. D., et al. 2002, A\&A, 394, 539

Jerzykiewicz, M., \& Molenda-Zakowicz, J. 2000, Acta Astron., 50, 369 Kovtyukh, V. V. 2007, MNRAS, 378, 617

Li Causi, G., Antoniucci, S., \& Tatulli, E. 2008, A\&A, 479, 589

Perryman, M. A. C., Lindegren, L., Kovalevsky, J., et al. 1997, A\&A, 323, 49 Petrov, R. G., Malbet, F., Weigelt, G., et al. 2007, A\&A, 464, 1

Richichi, A., Percheron, I., \& Khristoforova, M. 2005, A\&A, 431, 773

Smiljanic, R., Barbuy, B., de Medeiros, J. R., \& Maeder, A. 2006, A\&A, 449, 655

Tatulli, E., Millour, F., Chelli, A., et al. 2007, A\&A, 464, 29

Weiss, W. W. 1986, A\&A, 160, 243

Westbrook, O. W., Evans, N. R., Wolk, S. J., et al. 2008, ApJS, 176, 218

Xu, H. Y., \& Li, Y. 2004, A\&A, 418, 213 
A. Domiciano de Souza et al.: Diameter and photospheric structures of Canopus from AMBER/VLTI interferometry, Online Material $p$ I

Table 1. Log of the AMBER/VLTI observations of Canopus and the calibrator star (HD 79917) for each observing block. The mean projected base length $\left(B_{\text {proj }}\right)$ and position angle (PA) are given, together with the airmass (AM), and the coherence time measured in the visible $\left(\tau_{0}\right)$. The angular diameters for the calibrator are (Richichi et al. 2005): $1.54 \pm 0.02$ mas ( $H$ band) and $1.55 \pm 0.02$ mas ( $K$ band).

\begin{tabular}{|c|c|c|c|c|c|c|c|c|c|}
\hline Star & $\begin{array}{c}\text { Date/UT } \\
\text { (start of observation) }\end{array}$ & $\mathrm{AM}$ & $\begin{array}{l}\tau_{0} \\
\text { (s) }\end{array}$ & $\begin{array}{l}B_{\text {proj }}^{1} \\
(\mathrm{~m})\end{array}$ & $\begin{aligned} \mathrm{PA}^{1} \\
\left({ }^{\circ}\right)\end{aligned}$ & $\begin{array}{l}B_{\text {proj }}^{2} \\
(\mathrm{~m})\end{array}$ & $\begin{array}{c}\mathrm{PA}^{2} \\
\left({ }^{\circ}\right)\end{array}$ & $\begin{array}{l}B_{\text {proj }}^{3} \\
(\mathrm{~m})\end{array}$ & $\begin{array}{r}\mathrm{PA}^{3} \\
\left({ }^{\circ}\right)\end{array}$ \\
\hline Canopus & 2007-04-06T00:35:10 & 1.288 & 0.0034 & 70.3 & -132.2 & 90.0 & -37.6 & 109.7 & -77.3 \\
\hline HD 79917 & 2007-04-06T00:03:25 & 1.055 & 0.0035 & 89.5 & -160.0 & 86.9 & -72.4 & 127.4 & -117.0 \\
\hline Canopus & 2007-04-06T00:40:45 & 1.302 & 0.0039 & 69.7 & -131.6 & 89.9 & -36.4 & 108.8 & -76.1 \\
\hline HD 79917 & 2007-04-06T00:03:25 & 1.055 & 0.0035 & 89.5 & -160.0 & 86.9 & -72.4 & 127.4 & -117.0 \\
\hline Canopus & 2007-04-06T00:50:46 & 1.328 & 0.0037 & 68.5 & -130.4 & 89.8 & -34.3 & 107.0 & -73.9 \\
\hline HD 79917 & 2007-04-06T01:22:46 & 1.034 & 0.0034 & 87.3 & -150.0 & 90.4 & -60.2 & 126.0 & -104.1 \\
\hline Canopus & 2007-04-06T00:56:12 & 1.343 & 0.0032 & 67.9 & -129.8 & 89.7 & -33.1 & 106.0 & -72.6 \\
\hline HD 79917 & 2007-04-06T01:22:46 & 1.034 & 0.0034 & 87.3 & -150.0 & 90.4 & -60.2 & 126.0 & -104.1 \\
\hline Canopus & 2007-04-06T02:10:33 & 1.649 & 0.0029 & 57.1 & -121.5 & 88.3 & -15.8 & 91.3 & -52.8 \\
\hline HD 79917 & 2007-04-06T02:41:58 & 1.107 & 0.0025 & 82.7 & -142.1 & 88.6 & -47.0 & 115.7 & -92.4 \\
\hline Canopus & $2007-0$ & 1.263 & 0.0036 & 71.1 & -133.0 & 90.1 & -39.0 & 110.8 & -78.8 \\
\hline HD 79917 & 2007-04-06T23:42:02 & 1.073 & 0.0043 & 89.7 & -161.8 & 85.7 & -74.2 & 126.7 & -119.2 \\
\hline Canopus & 2007-04-07T00:38:36 & 1.306 & 0.0041 & 68.8 & -130.7 & 89.8 & -34.8 & 107.4 & -74.4 \\
\hline HD 79917 & 2007-04-07T01:16:10 & 1.033 & 0.0044 & 87.4 & -150.5 & 90.4 & -61.0 & 126.3 & -104.8 \\
\hline Canopus & 2007-04-07T01:50:12 & 1.563 & 0.0040 & 59.6 & -123.2 & 88.6 & -19.6 & 94.5 & -57.4 \\
\hline HD 79917 & 2007-04-07T02:35:39 & 1.103 & 0.0029 & 83.0 & -142.5 & 88.8 & -47.8 & 116.5 & -93.0 \\
\hline Canopus & 2007-04-07T02:05:56 & 1.645 & 0.0032 & 57.2 & -121.5 & 88.3 & -16.0 & 91.4 & -53.0 \\
\hline HD 79917 & 2007-04-07T02:35:39 & 1.103 & 0.0029 & 83.0 & -142.5 & 88.8 & -47.8 & 116.5 & -93.0 \\
\hline Canopus & 2007-04-08T00:46:06 & 1.337 & 0.0035 & 67.8 & -129.7 & 89.7 & -33.0 & 105.9 & -72.5 \\
\hline HD 79917 & 2007-04-07T23:59:52 & 1.052 & 0.0025 & 89.3 & -158.6 & 87.7 & -70.8 & 127.6 & -115.2 \\
\hline Canopus & 2007-04-08T01:03:12 & 1.389 & 0.0032 & 65.6 & -127.8 & 89.3 & -29.2 & 102.7 & -68.5 \\
\hline HD 79917 & 2007-04-08T01:36:48 & 1.044 & 0.0027 & 86.4 & -147.8 & 90.4 & -57.1 & 124.3 & -101.2 \\
\hline
\end{tabular}

